\title{
Doença Cardiovascular no Diabetes Mellitus: Análise dos Fatores de Risco Clássicos e Não-Clássicos
}

\begin{abstract}
RESUMO
A doença cardiovascular (DCV), incluindo a doença arterial coronariana (DAC), acidente vascular cerebral (AVC) e doença arterial periférica (DAP), é importante causa de morte em populações, especialmente na diabética. Indivíduos diabéticos apresentam risco aumentado de 3 a 4 vezes de sofrer evento cardiovascular e o dobro do risco de morrer deste evento quando comparados à população geral. Tem havido declínio na mortalidade por DCV, porém a queda nas mortes por DAC em portadores de diabetes tem sido bastante inferior à de não-diabéticos. Vários fatores presentes no diabetes favorecem a maior ocorrência de DCV, como a hiperglicemia, a resistência à insulina, além de fatores de risco clássicos e não-clássicos (hipertensão arterial sistêmica, dislipidemia, obesidade, estado inflamatório subclínico e outros). É possível que o potencial aterogênico da obesidade decorra em parte da produção aumentada de citocinas pelos adipócitos. Devido à marcante associação entre diabetes e DCV, e prognóstico desfavorável após um evento, é importante identificar quais são os indivíduos de mais alto risco e como rastreá-los. A American Heart Association e a American Diabetes Association recomendam estratificação do risco de pacientes sintomáticos por testes diagnósticos. O desafio está em identificar pacientes diabéticos assintomáticos que se beneficiariam de testes diagnósticos para detecção precoce de DCV, visando viabilizar medidas preventivas ou terapêuticas, capazes de reduzir morbi-mortalidade. O benefício do controle glicêmico e dos demais fatores de risco na prevenção de eventos CV no diabetes já documentado, justifica estabelecer estratégias que otimizem a identificação e possibilitem intervenções nos pacientes de alto risco, buscando reduzir mortalidade. (Arq Bras Endocrinol Metab 2007;51/2:257-267)
\end{abstract}

Descritores: Doença cardiovascular; Fatores de risco; Diabetes mellitus

\begin{abstract}
Cardiovascular Disease in Diabetes Mellitus: Classical and Non-Classical Risk Factors.

Cardiovascular disease, which includes coronary heart disease (CHD), cerebrovascular disease (CVD), and peripheral vascular disease (PVD), is the leading cause of mortality in populations, particularly in the diabetic one. Individuals with diabetes have at least a two-fold to four-fold increased risk of having cardiovascular events and a double risk of death compared with age-matched subjects without diabetes. A decline in mortality from CVD has been shown, but decline due to CHD is consistently lower in individuals with diabetes when compared with non-diabetics. The presence of several factors in diabetes leads to high occurrence of CVD such as hyperglicemia, insulin resistance, and classical and non-classical risk factors (systemic hypertension, dyslipidemia, obesity, proinflammatory condition and others). It is possible that the atherogenic role of obesity may be at least in part due to increased adipocyte production of cytokines. Considering the marked association of diabetes and CVD and unfavorable prognosis following an event, it is important to identify who is at high risk and how to screen. The American Heart Association and American Diabetes Association recommend risk stratification using diagnostic tests. However, the challenge is to accurately identify patients without a prior history of an event and those without symptoms strongly suggesting CVD, in whom additional testing would be indicated in order to achieve the most effective prevention. The benefits of glycemic control and the other risk factors have already been shown and justify optimization of the management of this high-risk population, aiming to reduce cardiovascular mortality disease and improve quality of life. (Arq Bras Endocrinol Metab 2007;51/2:257-267)
\end{abstract}

Keywords: Cardiovascular disease; Risk factors; Diabetes mellitus revisão

\author{
ANTONELA F.A. SIQUEIRA \\ BIANCA DE ALMEIDA-PITITTO \\ SANDRA R.G. FERREIRA
}

Disciplina de Endocrinologia,

Universidade Federal de

São Paulo - UNIFESP/EPM

(AFAS \& BA-P), e Departamento

de Nutrição, Faculdade de

Saúde Pública, Universidade de

São Paulo - USP (SRGF),

São Paulo, SP. 
$\mathrm{O}$ MPACTO DELETÉRIO DO DIAGNÓSTICO de diabetes mellitus (DM) na morbidade cardiovascular foi definitivamente comprovado em 1998, com a publicação de Haffner e cols. (1), apontando que sua presença conferia risco de evento coronariano isquêmico similar ao de indivíduos não-diabéticos sabidamente coronariopatas. Estes autores atribuíram ao DM tipo 2 o termo "equivalente coronariano", diante da verificação da mesma incidência de infarto agudo do miocárdio (IAM) em 7 anos de 20\%, tanto em indivíduos sem DM tipo 2 com IAM prévio como naqueles com DM tipo 2 que nunca haviam sofrido IAM. O estudo de Framingham já chamava atenção para o fato de que o DM dobra o risco de doença cardiovascular (DCV) em homens e triplica em mulheres (2). Aproximadamente 13\% dos pacientes com DM acima de 65 anos já tiveram um episódio de acidente vascular cerebral (AVC) (3). No que se refere à doença arterial periférica (DAP), afeta de 8 a 10 milhões de americanos com incidência crescente (4). Estudos epidemiológicos apontam o DM e o tabagismo como importantes fatores de risco para DAP, aumentando de 2 a 4 vezes o risco relativo $(5)$.

A DCV aterosclerótica constitui a principal causa de morte em diversas populações, sendo que o acometimento dos territórios arteriais coronariano, cerebral e dos membros inferiores são os que mais contribuem para a morbi-mortalidade dos portadores de DM. Indivíduos diabéticos apresentam o dobro do risco de morrer por causa cardiovascular quando comparados à população geral (6). Quando sofrem evento coronariano, têm maior risco de morte que aqueles sem a doença (7). A presença de DM também eleva em 3 vezes a mortalidade por AVC (8).

Dados populacionais norte-americanos revelaram que 58\% das mortes no ano de 2002 tiveram a DCV como causa principal ou contribuinte (9), apesar da tendência de queda observada nas últimas décadas (10), atribuída ao melhor controle de certos fatores de risco e à melhora no tratamento dessas doenças. Dados analisados por Lotufo mostram que a mortalidade pelas DCV no Brasil é das mais elevadas quando comparada à de outros países. Apesar de as mortes por esta causa serem mais freqüentes no sexo masculino, a mortalidade das mulheres brasileiras por DCV é maior do que em outras regiões do mundo. Tem-se verificado um declínio consistente nas taxas de mortalidade cardiovascular no Estado de São Paulo desde o início dos anos 80. Em cidades como Porto Alegre e Curitiba, a situação é de estabilidade, enquanto que no Rio de Janeiro tem sido observado certo aumento nas taxas (11). Infelizmente, este declínio não vem sendo relata- do de maneira impactante nos indivíduos diabéticos. Uma comparação de dados de uma coorte norte-americana do período de 1971 a 1975 com uma mais recente (1982 a 1984) revelou que, em homens nãodiabéticos, houve $44 \%$ de diminuição na mortalidade por doença isquêmica do coração, enquanto que nos portadores de DM esta queda foi de apenas 17\%. Para as mulheres, a situação é ainda mais grave, uma vez que, enquanto houve $20 \%$ de declínio na mortalidade por doença isquêmica do coração nas não-diabéticas, houve aumento de $11 \%$ nas portadoras de DM (12).

O DM tipo 2 freqüentemente associa-se a outras anormalidades, tais como a obesidade visceral, a hipertensão arterial sistêmica (HAS) e a dislipidemia. A síndrome metabólica (SM) - conjunto de anormalidades que aumentam o risco cardiovascular — está associada à elevação da mortalidade, tanto cardiovascular como geral (13). Nos EUA, está presente em $24 \%$ da população adulta (14). Em relação à mortalidade por doença arterial coronariana, a presença de um ou dois componentes da SM já aumenta em duas vezes o risco de morte, e o impacto do DM tipo 2 nestes indivíduos é evidente: hazard ratio $=1,65$ naqueles com SM mas sem DM tipo 2 vs. 2,87 naqueles com SM e com DM tipo 2 (14). Um estudo populacional conduzido em nipo-brasileiros exemplifica a contribuição da SM e do DM tipo 2 na ocorrência de doença macrovascular: houve diferença significante entre a prevalência de DCV nos indivíduos com e sem a SM (16,9\% vs. 11,2\%), e aqueles com DM tipo 2 apresentaram quase o dobro da prevalência de DCV ( razão de prevalência $=1,91)(15)$.

Indivíduos diabéticos do tipo 1 também apresentam prevalência aumentada de DCV, sendo 10 vezes maior que em controles não-diabéticos da mesma faixa etária (16). Em uma coorte inglesa com mais de 23.000 indivíduos diabéticos tipo 1 , a doença isquêmica do coração foi responsável por $8 \%$ das mortes em homens e $11 \%$ em mulheres com menos de 40 anos. Os homens com DM tipo 1 morreram mais que os não-diabéticos, e a taxa de mortalidade por coronariopatia foi maior em mulheres diabéticas do que em mulheres e homens nãodiabéticos (17), chamando a atenção para o impacto do DM tipo 1 na mortalidade também de indivíduos jovens, principalmente do sexo feminino. Nesta mesma coorte foi avaliada a mortalidade cerebrovascular destes pacientes, revelando que $4 \%$ das mortes em indivíduos com menos de 40 anos foram decorrentes de acidente vascular cerebral (AVC), principalmente de causa isquêmica (18). A título de comparação, na população geral adulta norte-americana, apenas $2 \%$ haviam sofrido um AVC no último censo (9). 
Recentemente, o estudo INTERHEART mostrou que o risco populacional de IAM atribuível ao DM foi de cerca de 10\%, considerando diferentes regiões geográficas e etnias (19). Diante das projeções de prevalência crescente do DM nas populações $(20)$ e de seu forte impacto na morbidade e mortalidade especialmente cardiovascular, prevenir e tratar o DM são de fundamental importância em termos de saúde pública.

\section{MARCADORES DE HIPERGLICEMIA E DCV}

Habitualmente, o metabolismo glicídico vem sendo estudado com base nos valores da glicemia de jejum (GJ), glicemia de 2 horas pós-sobrecarga oral de glicose (G2h) e hemoglobina glicada. Estudos recentes revelam que o risco cardiovascular é gradual e progressivo à medida que a GJ, G2h e a hemoglobina glicada se elevam, ainda em níveis pré-diabéticos (21). No entanto, vale lembrar que os pontos de corte para GJ e G2h que definem DM foram baseados no nível a partir do qual a ocorrência da retinopatia aumenta significantemente (22), sem considerar a predição do risco cardiovascular (23). Atenta a este fato, em 2003 a American Diabetes Association (ADA) reduziu os valores de GJ para $100 \mathrm{mg} / \mathrm{dL}$ (24).

Estudos revelam elevação significativa do risco de morte por todas as causas, por DCV e por coronariopatia a partir do valor de $126 \mathrm{mg} / \mathrm{dl}$ para GJ (2l). No entanto, uma limitação da GJ como parâmetro diagnóstico é que ela não aumenta com a idade, reconhecido fator de risco para intolerância à glicose e DCV. Desse modo, a determinação exclusiva da GJ pode ser menos sensível do que a G2h em indivíduos idosos (21). Usando os critérios diagnósticos da ADA, de 1997, detectou-se $7 \%$ de alteração no metabolismo glicídico (glicemia de jejum alterada: GJ > $110 \mathrm{mg} / \mathrm{dL}$ ) em população americana adulta. Esse valor passou a ser mais que o dobro nos indivíduos entre 40 e 74 anos, quando a G2h foi levada em consideração (tolerância à glicose diminuída: G2h > 140 e $<200 \mathrm{mg} / \mathrm{dl}$ ), efeito este decorrente da tendência de a G2h se elevar com a idade (25). A prevalência de hiperglicemia pós-sobrecarga com normoglicemia de jejum foi estimada em $48 \% \mathrm{em}$ homens e $70 \%$ em mulheres americanas com mais de 65 anos de idade e sem diagnóstico prévio de DM tipo 2 $(26,27)$. Depreende-se que a proposta da ADA para estabelecer o estado de tolerância à glicose com base exclusivamente na GJ pode estar deixando de identificar indivíduos com risco cardiovascular aumentado.
Em diversos estudos prospectivos, indivíduos com teste oral de tolerância a glicose (TOTG) alterado apresentaram aumento na mortalidade total e cardiovascular quando comparados àqueles normotolerantes à glicose (21). O estudo DECODE, envolvendo diversas populações européias, representa provavelmente o marco mais importante na identificação da G2h como sendo mais fiel e sensível na predição de morte cardiovascular. Esses autores advogam que o TOTG oferece informação prognóstica adicional, permitindo a identificação de indivíduos com elevação da G2h, à qual se associa maior risco de morte, para qualquer nível de GJ (28). Tal associação independente entre mortalidade e G2h foi também documentada na população americana de Framingham (29), porém este efeito se mostrou mais forte quando na presença de GJ igualmente alterada (27). O papel da hiperglicemia pós-sobrecarga para o risco cardiovascular tem sido alvo de grande discussão na literatura, e a necessidade do TOTG para melhorar a predição deste risco ainda tem sido questionada.

Uma maior importância vem sendo dada à hiperglicemia como fator de risco para a micro do que para a macroangiopatia, visto que cada aumento percentual da hemoglobina glicada eleva em $70 \%$ o risco de retinopatia, $20 \%$ o de proteinúria e $10 \%$ o de complicações macrovasculares (30), porém $70 \%$ das mortes em indivíduos diabéticos são decorrentes de eventos cardiovasculares (31). Uma meta-análise que avaliou o impacto da hemoglobina glicada na DCV em indivíduos diabéticos, mostrou que no DM tipo 1 cada ponto percentual de aumento na hemoglobina glicada confere um risco relativo de 1,15 para DAC e de 1,32 para DAP. No DM tipo 2, o risco relativo foi de 1,18 para uma composição de desfechos cárdio e cerebrovasculares e de 1,28 para a $\operatorname{DAP}(6)$.

Entre os mecanismos fisiopatológicos pelos quais a hiperglicemia resulta numa aceleração da aterotrombogênese, estão a glicação de lipoproteínas, prolongando a meia-vida da lipoproteína de baixa densidade (LDL), facilitando sua oxidação e aumentando seu poder de agressão ao endotélio, além da formação de outros produtos finais de glicação, que promovem a disfunção endotelial generalizada (31). No entanto, a hiperglicemia leve per se poderia ser encarada como indicativa de risco quando outras alterações metabólicas estão contribuindo para a aterosclerose nos estados de resistência à insulina (RI). 


\section{RESISTÊNCIA À INSULINA E DCV}

A aterosclerose está mais relacionada com a duração e gravidade da RI do que com a hiperglicemia em si. A RI associa-se com disfunção endotelial, e foi verificada melhora na função vasomotora tanto com metformina (32) quanto com tiazolidinedionas (33) em pacientes resistentes à insulina. A pró-insulina, que está elevada na RI, aumenta a síntese de inibidor do ativador do plasminogênio (PAI-1), prejudicando a fibrinólise (34). Além disso, no Quebec Cardiovascular Study, que seguiu por 5 anos uma amostra de homens de meia-idade, níveis aumentados de insulina plasmática no jejum, associada à dislipidemia da SM (apolipoproteína B aumentada e presença de partículas de LDL pequenas e densas), aumentaram o risco de evento coronariano em 20 vezes (35).

Outro ponto controverso é se a hiperinsulinemia seria fator causal de DCV ou apenas um marcador de RI. Sabe-se que a insulina apresenta atividade mitogênica e promove proliferação de células musculares lisas em concentrações suprafisiológicas. Ela também induz o acúmulo intracelular de ésteres de colesterol e dificulta o efluxo de colesterol mediado pela lipoproteína de baixa densidade (HDL). A literatura é controversa e a causalidade ainda não foi provada, mas parece que a hiperinsulinemia compensatória associase com DCV porque ambas emergem do solo comum da RI (36).

\section{FATORES DE RISCO CARDIOVASCULAR NO DIABETES MELLITUS}

Os portadores de DM tipo 2 comumente apresentam outros fatores de risco cardiovascular ligados à RI. Historicamente, o Framingham Heart Study definiu os fatores de risco clássicos como sendo DM, HAS, hipercolesterolemia, tabagismo e os não-modificáveis, como idade, sexo masculino e história familiar de DCV (37). O escore de risco cardiovascular de Framingham, amplamente utilizado na predição de eventos cardiovasculares, baseia-se nestes fatores, porém desconsiderando a história familiar e acrescentando uma pontuação para $\mathrm{o}$ HDL baixo (38). Na atualidade, há uma intensa busca por novos marcadores que possam refinar essa predição de eventos, principalmente nos indivíduos sabidamente de alto risco, porém com um impacto ainda frustro naquela já conferida pelos fatores clássicos. $\mathrm{O}$ Atherosclerosis Risk In Communities Study (ARIC) avaliou o incremento no poder preditivo de risco cardiovascular resultante da inclusão da proteína $\mathrm{C}$ reativa $(\mathrm{PCR})$ e outros fatores não-clássicos nos atuais modelos de predição, com base em análise de curva ROC, não encontrando aumento significante na área sob a curva; os autores apenas reforçaram a importância dos fatores de risco "maiores" e modificáveis na identificação de indivíduos de risco que se beneficiariam de medidas de prevenção (39). A seguir, a importância dos outros componentes da SM e demais fatores de risco clássicos e não-clássicos nos indivíduos com DM serão discutidos individualmente.

\section{Dislipidemia}

O fenótipo lipídico aterogênico presente na SM é resultado do excesso de tecido adiposo visceral, que, devido à intensa atividade lipolítica, libera grandes quantidades de ácidos graxos livres na circulação. Em conseqüência, há menor depuração hepática de insulina e hiperinsulinemia sistêmica, redução na degradação da apolipoproteína $B$ (ApoB) e maior secreção hepática de lipoproteínas de muito baixa densidade (VLDL). Isso resulta em maior geração de LDLs pequenas e densas, diminuição do colesterol na HDL e aumento dos triglicérides (40). Apenas as duas últimas alterações fazem parte dos parâmetros diagnósticos de SM sugeridos pelo National Cholesterol Education Program (NCEP) (41); no entanto, a LDL pequena e densa vem sendo apontada como importante fator de risco para a doença coronariana. Resultados do Familial Atherosclerosis Treatment Study mostraram que a diminuição na densidade das partículas de LDL, induzida pelo tratamento agressivo dos lipídios séricos, associou-se melhor com a regressão da estenose coronariana do que a mudança nos níveis séricos da LDL (42). Seja qual for o grau de dislipidemia, os indivíduos diabéticos têm mais DCV que os não-diabéticos, pois a glicação da LDL modifica a partícula, aumenta sua carga elétrica negativa e facilita sua oxidação, tornando-a mais aterogênica. Nesse processo, há conjugação não-enzimática da ApoB com glicose, e a partícula formada não é reconhecida pelo receptor de LDL, mas sim por receptores scavenger de macrófagos da parede arterial, induzindo a formação de células espumosas (43).

A dislipidemia da SM costuma aparecer antes do diagnóstico de DM tipo 2, sendo que a elevação dos triglicérides é a alteração mais freqüente (44). A determinação do colesterol não-HDL é um forte preditor de DCV nos indivíduos diabéticos (45), mas a LDL continua sendo o principal alvo terapêutico. No DM tipo l, o perfil lipídico é amplamente relacionado ao controle glicêmico, sendo que a hipertrigliceridemia e o HDL baixo surgem em indivíduos descompensados (46).

O NCEP propôs alvos mais rigorosos para o nível sérico de LDL quando o indivíduo é portador de $\mathrm{DM}(\mathrm{LDL}<100 \mathrm{mg} / \mathrm{dL})$, baseando-se no fato de este 
ser "equivalente coronariano", segundo sugerido por Haffner e cols. (1). Em estudos de prevenção secundária de DCV através do tratamento agressivo dos lipídios com estatinas $(47,48)$, foi verificado que em subgrupos de indivíduos com DM houve redução da recorrência de eventos cardiovasculares agudos naqueles que atingiram os alvos mais rigorosos do LDL-colesterol, sem aumentar o risco de morte por outras causas.

\section{Hipertensão arterial sistêmica}

A HAS é diagnosticada em 40-60\% dos indivíduos com DM tipo 2 entre 45 e 75 anos (49), e em muitos já está presente ao diagnóstico. No DM tipo 1, a HAS não costuma ocorrer enquanto não houver sinais e sintomas de doença renal. Dados do United Kingdom Prospective Diabetes Study (UKPDS) revelaram que cada redução de $10 \mathrm{mmHg}$ na pressão arterial sistólica (PAS) traz uma redução de $12 \%$ na ocorrência de qualquer complicação relacionada ao DM tipo 2, inclusive DCV. O menor risco foi identificado quando a PAS mantinha-se abaixo de $120 \mathrm{mmHg}$. A incidência de IAM no grupo com PAS média de $160 \mathrm{mmHg}$ foi de 33 por 1.000 pessoas/ano, e caiu para 18 no grupo com PAS média de $120 \mathrm{mmHg}$ (50). Em face destas e de outras observações, o tratamento agressivo da pressão arterial (PA) deve ser prioridade nos pacientes diabéticos, com um alvo terapêutico de $130 \times 80$ $\mathrm{mmHg}(51)$.

Grandes estudos prospectivos já avaliaram o impacto do tratamento da HAS na ocorrência de eventos cardiovasculares, em subgrupos portadores de DM. No estudo Heart Outcomes Prevention Evaluation (HOPE), a análise de subgrupo de pacientes diabéticos revelou que o inibidor da enzima conversora da angiotensina ramipril reduziu em $25 \%$ o risco dos desfechos cardiovasculares (IAM, AVC ou morte cardiovascular), em $37 \%$ o de mortalidade cardiovascular e em $24 \%$ o de mortalidade total. Mesmo após ajuste para as mudanças na PA, o ramipril reduziu o risco de desfechos cardiovasculares, sugerindo uma ação cardio e renoprotetora deste medicamento em indivíduos com DM (52). Os antagonistas da angiotensina II também apresentam tal benefício, independentemente da redução da PA. No estudo Losartan Intervention For Endpoint reduction in hypertension (LIFE), o losartan foi mais eficaz que o atenolol na redução da morbi-mortalidade cardiovascular e mortalidade por todas as causas em pacientes com HAS, DM e hipertrofia ventricular esquerda (53).

\section{Hábitos de vida}

\section{Tabagismo}

Assim como em indivíduos não-diabéticos, o fumo aumenta a morbi-mortalidade cardiovascular (54), mas este aumento no risco volta gradualmente para o normal 10 anos após a cessação do hábito de fumar (55). O cigarro também aumenta a concentração de colesterol na VLDL e diminui na HDL, além de piorar o controle glicêmico, uma vez que induz RI (56).

\section{Consumo de álcool}

O consumo moderado de bebidas alcoólicas parece ter um efeito benéfico nos sistema circulatório. Em um seguimento de 5,5 anos de 2.790 pacientes diabéticos no Physicians' Health Study, aqueles que consumiram álcool diária ou semanalmente tiveram menor risco de morte por DAC do que aqueles que nunca ou raramente bebiam (57). Conclusões do estudo INTERHEART apontam 9 fatores de risco facilmente mensuráveis e modificáveis, responsáveis por mais de $90 \%$ dos IAM. Entre eles está a ausência do consumo leve a moderado regular de álcool.

\section{Sedentarismo}

A grande maioria das evidências de que o exercício físico traz benefícios cardiovasculares provém de estudos observacionais, sendo provável que o hábito de se exercitar seja apenas um dos componentes na adoção de um estilo de vida mais saudável, dificultando a análise do seu impacto isoladamente. No entanto, alguns dados relevantes merecem destaque. Numa coorte de quase 3.000 diabéticos, aqueles que andaram por no mínimo 2 horas na semana tiveram menor taxa de mortalidade, comparados com indivíduos inativos (58); em outra coorte com mais de 3.000 diabéticos da Finlândia, a atividade física ocupacional e de lazer associou-se com redução significante da mortalidade geral e por DCV (59).

\section{Microalbuminúria e insuficiência renal}

A presença de microalbuminúria (MA) tem sido interpretada como indicativa de disfunção endotelial generalizada. Microalbuminúria associa-se com elevação de risco cardiovascular tanto em populações diabéticas (60) como em não-diabéticas $(61,62)$. Uma correlação linear independente de MA com morbi-mortalidade cardiovascular inicia-se ainda em valores normais de excreção de albumina (63). Nos nipo-brasileiros, a razão albumina-creatinina urinária correlacionou-se com PAS e estado de tolerância a glicose (64) e com 
doença macrovascular (15). Numa revisão de cerca de 9.000 participantes do estudo HOPE, a presença de microalbuminúria associou-se com maior risco relativo de desfechos cardiovasculares (IAM, AVC e morte cardiovascular) em indivíduos com e sem DM (1,97 e 1,61 respectivamente) (62). Resultados do estudo LIFE também ilustram esse impacto. Para cada aumento de 10 vezes na razão albumina-creatinina urinária, o risco de morte cardiovascular, IAM ou AVC aumentou em 39\% e o risco de morte cardiovascular, em $47 \%$ nos pacientes diabéticos (65).

O rim é referido como o "órgão sensor de risco cardiovascular". No que diz respeito à função renal, uma análise do UKPDS revelou aumento importante na taxa de mortalidade cardiovascular anual com a progressão da nefropatia diabética. Essa taxa foi de 0,7\% nos indivíduos livres de doença, subindo para 2,0\% em microalbuminúricos, $3,5 \%$ em macro e finalmente $12,1 \%$ naqueles com insuficiência renal crônica estabelecida $(50)$.

\section{Obesidade e marcadores inflamatórios}

A obesidade coexiste com o DM tipo 2 na grande maioria dos pacientes. O tecido adiposo vem sendo considerado um grande órgão endócrino, na medida em que os adipócitos secretam hormônios e uma gama de citocinas inflamatórias que participam do processo aterogênico. A circunferência da cintura, como um parâmetro de avaliação de obesidade visceral, foi considerada pela International Diabetes Federation o principal critério na definição de SM, pela praticidade de sua medição e forte associação com RI (66); adicionalmente, a razão cintura-quadril mostrou associação significante com risco de IAM em vários grupos étnicos. No entanto, a contribuição do índice de massa corporal (IMC) para o risco cardiovascular, independentemente dos outros componentes da SM, permanece controversa. Um estudo conduzido em mulheres menopausadas saudáveis encontrou associação significante da aterosclerose carotídea com SM, mas não com a obesidade definida pelo IMC (67). Continua sendo prudente a recomendação de perda de peso, porém o controle das demais anormalidades metabólicas, inclusive no perfil glicêmico, deve ser considerado o alvo primário de tratamento nessa população.

É possível que o potencial aterogênico da obesidade possa ser em parte decorrente da secreção de citocinas inflamatórias. Estima-se que $25 \%$ da interleucina-6 (IL-6) do organismo seja produzida no tecido adiposo (68), sendo ela um dos principais estímulos para a produção hepática de proteína $\mathrm{C}$ reativa (PCR) (69). Certos autores acreditam que a associação da PCR com doença aterosclerótica seja decorrente da sua forte relação com obesidade. Um estudo conduzido na população do NHANES III verificou associação de sobrepeso e obesidade com níveis séricos elevados de PCR; também a razão cintura-quadril associou-se à PCR, independentemente do IMC, ressaltando a importância da adiposidade abdominal (69).

Diversos estudos com esses marcadores reforçaram a hipótese de ser a aterosclerose uma doença inflamatória crônica (70). Altos níveis de IL-6 foram associados a risco de IAM (71). Em seguimentos prospectivos de homens saudáveis, os níveis de PCR foram apontados como fator de risco para doença coronariana, DAP e até para morte súbita $(72,74)$. O Women Health Study mostrou que, em mulheres menopausadas, também houve forte associação da PCR com risco de eventos agudos (75). Há poucos anos, a PCR foi considerada um preditor de eventos cardiovasculares mais forte que o LDL, e foi sugerido que adicionaria informação prognóstica ao escore de risco de Framingham (76). Mais recentemente, no entanto, este fato vem sendo contestado e, no estudo ARIC, a PCR não adicionou muito poder nos modelos de predição de risco cardiovascular já consagrados (39).

A população diabética, por já apresentar risco elevado de eventos isquêmicos, poderia se beneficiar de um possível refinamento na predição conferido pela PCR. No entanto, em um estudo conduzido em índios americanos, a PCR não foi preditiva de eventos no subgrupo portador de DM (77).

\section{RASTREAMENTO DE DOENÇA CARDIOVASCULAR NO INDIVÍDUO DIABÉTICO}

A detecção precoce de DCV pode viabilizar medidas preventivas ou terapêuticas, potencialmente capazes de reduzir morbi-mortalidade. É importante ressaltar que, mesmo se o indivíduo com DM apresentar testes de rastreamento para DCV negativos, este continua sendo considerado de risco comparável ao daqueles não-diabéticos com DCV estabelecida, devendo receber conduta agressiva no controle dos outros fatores de risco (78).

\section{Doença coronariana}

É conhecido que indivíduos com DM apresentam IAM silencioso com maior freqüência, assim como mais complicações pós-IAM (insuficiência cardíaca e neuropatia autonômica cardíaca), atribuídas ao acometimento difuso dos vasos coronarianos no $\operatorname{DM}(79)$. 
A American Diabetes Association (ADA) (80) recomenda que indivíduos com sintomas de coronariopatia típicos ou atípicos ou ECG de repouso com alterações sugestivas de isquemia ou IAM deveriam ser submetidos a ecocardiograma ou cintilografia miocárdica sob estresse, por exercício ou farmacológico. Se forem assintomáticos, mas com idade maior que 35 anos, sedentários e estarem planejando iniciar exercício físico, ou apresentarem evidência de arteriopatia periférica ou carotídea ou pelo menos 2 fatores de risco (hipertensão, dislipidemia, tabagismo, história familiar e albuminúria), o teste ergométrico é o exame de escolha. Se o ECG for normal e apresentar, no máximo, um fator de risco, recomenda-se seguimento de rotina e ECG anual. Tal posicionamento da ADA foi reforçado em 2002 diante das French Guidelines, American College of Cardiology e American Heart Association da não realização de testes sob estresse físico ou farmacológico em indivíduos assintomáticos (78).

O valor preditivo negativo do teste ergométrico (TE) é alto, principalmente quando avaliação prévia identifica o indivíduo como de baixo risco $(81,82)$. Se positivo, o indivíduo deve ser submetido a ecocardiograma ou cintilografia miocárdica (MIBI) sob estresse (80). Se os exames de imagem sob estresse mostrarem alteração discreta ou ausência de anormalidades, esse indivíduo deve repetir o ecocardiograma ou MIBI a cada 2 anos; se alterados, estará indicada angiografia coronariana. Quando o TE for altamente sugestivo de coronariopatia, mesmo se assintomáticos, a cateterização coronariana está indicada (80). O cateterismo cardíaco e a arteriografia coronariana são capazes de detalhar a localização e a extensão das lesões, fornecendo subsídios para a estratégia terapêutica.

Embora indivíduos diabéticos apresentem acometimento coronariano mais difuso e acometendo as artérias pequenas e mais distalmentes, suas placas não diferem das placas encontradas em coronariopatas sem DM (80). A calcificação das placas permite que sejam detectadas por tomografia computadorizada (Electron Beam Computed Tomography - EBCT ou Tomografia Computadorizada Multislice); este exame é de baixa especificidade, sendo mais indicado para indivíduos de risco intermediário (83). Ausência de calcificação indica probabilidade de estenose inferior a 1\% (84). Escore de cálcio de 1-99 AU indica risco leve de coronariopatia (razão de odds de 2,1, comparado a escore de 0 ), enquanto de 100-399 AU risco moderado e $\geq 400 \mathrm{AU}$, grave $(85)$.

\section{Doença carotídea e cerebrovascular}

Poucos estudos avaliaram a doença cerebrovascular e carotídea especificamente em portadores de DM e, na maioria das vezes, são extrapolados da população geral.

Indivíduos com suspeita de doença carotídea por exame clínico devem ser investigados com ultrassonografia Doppler de carótida (USDC). A AHA alerta que os valores de espessura da camada íntima-média da carótida, medida pelas imagens do US modo $\mathrm{B}$, não devem ser extrapolados para pacientes com DM que, de base, já apresentam maior espessura da parede arterial (78). Indivíduos com estenose $<50 \%$ devem ser seguidos anualmente. Se a USDC mostrar estenose $>50 \%$, a investigação deve prosseguir com a angiografia por ressonância magnética (ARM), capaz de avaliar componentes das placas carotídeas extracranianas e identificar vulnerabilidade antes de serem rompidas (78). Na impossibilidade da ARM ou discordância com o resultado da USDC, é usada a angiografia por tomografia computadorizada, que proporciona visão ampla do leito vascular e tecidos adjacentes, capaz de reconstituir tridimensionalmente o lúmen vascular, aumentando a especificidade do diagnóstico (86). A importância deste exame baseia-se no fato de a principal lesão cerebrovascular no indivíduo diabético ocorrer em pequenas artérias paramediais penetrantes (79).

A angiografia é o método padrão-ouro, porém está relacionada a risco de complicações ou mesmo morte, o que restringe seu uso no rastreamento desta doença (87). Levando em consideração risco-benefício do exame, é ideal que o indivíduo seja investigado previamente quanto à alta probabilidade de doença carotídea por exames não-invasivos.

\section{Doença arterial periférica de membros inferiores}

A doença arterial periférica (DAP) no indivíduo diabético tende a aparecer de forma mais súbita e apresentar-se como acometimento mais difuso e distal das artérias de membros inferiores, quando comparada àqueles sem diabetes $(80)$.

$\mathrm{O}$ índice tornozelo-braço (ITB) - definido como a razão entre as pressões sistólicas da perna e do braço - é um método não-invasivo, objetivo e reprodutível, que permite evidenciar diagnóstico e gravidade da DAP. O ITB obtido pelo Doppler vascular apresenta sensibilidade em torno de $95 \%$ e especificidade de 99\%; se for usado o aparelho oscilométrico para aferição de pressão arterial sem o Doppler, esses percentuais reduzem-se para $88 \%$ de sensibilidade e $85 \%$ de especificidade na população geral (88). A ADA 
recomenda que seja feito em indivíduos diabéticos com sinais e sintomas de DAP, nos assintomáticos com $>50$ anos de idade, ou de qualquer idade desde que seja portador de dois ou mais fatores de risco para DCV, ou que tenha diagnóstico de DM há mais de 10 anos. Valores de ITB entre 0,91 e 1,3 são considerados normais, de 0,70 a 0,90 indica obstrução discreta, de 0,4 a 0,69 obstrução moderada, < 0,4, obstrução grave e > 1,4 indica artérias não-compressíveis (89). Considerando que pacientes com DM apresentam maior predisposição à calcificação das lesões e enrijecimento das artérias, o ITB pode estar falsamente elevado $(80)$.

O indivíduo com suspeita de DAP pode ainda ser submetido a teste ergométrico com aferição de pressão arterial em membros inferiores, avaliação laboratorial de pressão segmentar e volume de pulso e medida transcutânea de pressão parcial de oxigênio que podem fornecer informações quanto à localização e gravidade (80).

Em exames positivos para DAP nos quais é aventada a revascularização, a localização exata da lesão é fornecida pelos exames de imagem: o ultrasom duplex, o angiograma por ressonância magnética (RNM) e a angiografia. A angiografia é o exame padrão ouro, mas é invasivo e há a possibilidade de nefrotoxicidade em virtude do uso de contraste.

\section{O CONTROLE GLICÊMICO REDUZ O RISCO CARDIOVASCULAR?}

O UKPDS foi o mais importante estudo que avaliou o impacto do controle glicêmico na prevenção das complicações crônicas do DM tipo 2. Neste estudo, o tratamento intensivo com insulina ou sulfoniluréias não reduziu significativamente o número de eventos cardiovasculares, mas uma sub-análise sugeriu que a redução da hemoglobina glicada em 1\% associava-se com uma redução de $18 \%$ na ocorrência de IAM e de 15\% na de AVC (90). A metformina foi a medicação que trouxe os melhores resultados no que se refere à prevenção de DCV. O grupo de tratamento intensivo com metformina apresentou uma redução de risco de $42 \%$ nas mortes relacionadas ao DM e de $36 \%$ na mortalidade por todas as causas. Comparando metformina com sulfoniluréia ou insulina dentro do grupo de tratamento intensivo, ela mostrou-se mais eficaz na redução da mortalidade por todas as causas e por AVC, além de oferecer como vantagem o menor ganho de peso e menor incidência de hipoglicemias (91).

Diante do frustro impacto do controle glicêmico na redução da $\mathrm{DCV}$ em indivíduos diabéticos do tipo 2, encontrado no UKPDS, veio à tona a discussão se a intervenção nos múltiplos fatores de risco cardiovascular presentes no paciente diabético seria eficaz em melhorar nossa capacidade de prevenir eventos cardiovasculares. O estudo STENO II veio responder essa pergunta. Eles compararam o efeito de um tratamento intensivo, dirigido aos múltiplos fatores de risco cardiovascular, com alvos rigorosos pré-definidos, na ocorrência de desfechos cardiovasculares (IAM nãofatal, AVC não-fatal, revascularização, amputações e mortes de origem cardiovascular), em pacientes com DM tipo 2 e microalbuminúria. Os alvos deste estudo foram: pressão arterial menor que 130 x $80 \mathrm{mmHg}$; colesterol total menor que $175 \mathrm{mg} / \mathrm{dL}$; hemoglobina glicada menor que $6,5 \%$ e triglicérides menor que 150 $\mathrm{mg} / \mathrm{dL}$. Além disso, todos os pacientes alocados para o tratamento intensivo deveriam estar usando um inibidor da enzima conversora, independentemente do valor da pressão arterial e de um anti-agregante plaquetário. Para atingir estes alvos, eles lançaram mão de medidas não-farmacológicas e de medicações que foram sendo adicionadas conforme necessário. $\mathrm{O}$ seguimento foi de 8 anos e a redução de risco encontrada, da ordem de 50\% (92).

Agonistas do PPAR $\gamma$, que controlam os níveis glicêmicos por melhorar a sensibilidade periférica à insulina, poderiam ser agentes interessantes para atenuar o risco cardiovascular de indivíduos com SM. O PROspective pioglitAzone Clincal Trial In macroVascular Events (PROactive) selecionou pacientes de alto risco (indivíduos diabéticos com evidência de DCV), nos quais a pioglitazona foi comparada ao placebo quanto à sua capacidade de reduzir a ocorrência de eventos cardiovasculares. Houve diminuição significativa no desfecho secundário, pré-definido como morte por todas as causas, incidência de IAM não-fatal ou AVC, nos indivíduos que receberam pioglitazona em relação àqueles que receberam placebo (hazard ratio $=$ $0,84,0,72-0,98, \mathrm{p}=0,027)$. A grande crítica a este trabalho foi que o desfecho primário (tempo até a ocorrência de um novo evento macrovascular ou morte) não foi significantemente diferente entre os que receberam a droga e aqueles que receberam placebo (93).

O Diabetes Control and Complications Trial (DCCT), por sua vez, foi o principal estudo que demonstrou os benefícios do controle glicêmico intensivo na redução das complicações crônicas do DM tipo 1. A coorte que inicialmente participou do DCCT foi seguida por mais 11 anos pelo Epidemiology of Diabetes Interventions and Complications (EDIC), com o intuito de avaliar o impacto do tratamento intensivo realizado por 6,5 anos na diminuição do risco cardiovas- 
cular a longo prazo. Os autores concluíram que houve uma redução de $57 \%$ no risco de um evento cardiovascular grave, definido como IAM não-fatal, AVC ou morte por DCV, comparando o grupo de tratamento intensivo com o convencional. A eficácia de um período de controle glicêmico rigoroso na redução da DCV em longo prazo pode ser decorrente da diminuição da formação de produtos avançados da glicação, ou da redução das microangiopatias, uma vez que tanto microalbuminúria como neuropatia autonômica podem ser consideradas como fatores de risco cardiovascular (16).

De posse dos resultados mais recentes (STENO II, EDIC, PROactive) revelando o impacto do controle glicêmico e dos demais fatores de risco clássicos na prevenção de eventos no portador de DM, fica clara a urgência e a importância da intervenção agressiva, multifatorial, na busca de reduzir a morbi-mortalidade e melhorar a qualidade de vida destes pacientes de alto risco para a doença cardiovascular aterosclerótica.

\section{REFERÊNCIAS}

1. Haffner SM, Lehto S, Ronnemaa T, Pyorala K, Laakso M. Mortality from coronary heart disease in subjects with type 2 diabetes and in nondiabetic subjects with or without prior myocardio infarction. N Engl J Med 1998;339:229-34.

2. Kannel WB, McGee DL. Diabetes and cardiovascular risk factors: the Framingham Study. Circulation 1979;59:8-13.

3. Kuller LH, National Diabetes Data Group. Stroke and diabetes. In: Diabetes in America. Bethesda: National Institutes of Health/National Institute of Diabetes/Digestive and Kidney Diseases, 1995. p. 449-56.

4. Weitz JI, Byrne J, Clagett GP, Farkouh ME, Porter JM, Sackett $\mathrm{DL}$, et al. Diagnosis and treatment of chronic arterial insufficiency of the lower extremities: a critical review. Circulation 1996;94:3026-49.

5. Dormandy JA, Rutherford RB. Management of PAD. TASC WorkingGroup. TransAtlantic Inter-Society Consensus. J Vasc Surg 2000;31:S1-296.

6. Selvin E, Marinopoulos S, Berkenblit G, Rami T, Brancati FL, Powe NR, et al. Meta-analysis: glycosylated hemoglobin and cardiovascular disease in diabetes mellitus. Ann Int Med 2004;141(6):421-31.

7. Smith JW, Marcus FI, Serokman R. Prognosis of patients with diabetes mellitus after acute myocardial infarction. Am J Cardiol 1984;54:718-21.

8. Stamler J, Vaccaro O, Neaton JD, Wentworth D. Diabetes, other risk factors, and 12-year cardiovascular mortality for men screened in the Multiple Risk Factor Intervention Trial (MRFIT). Diabetes Care 1993;16:434-44.

9. Thom T, Haase N, Rosamond W, Howard VJ, Rumsfeld J, Manolio T, et al. Heart disease and stroke statistics-2006 update: a report from the American Heart Association Statistics Committee and Stroke Statistics Subcommittee. Circulation 2006;113:e85-151.

10. Rosamond WD, Chambless LE, Folsom AR, Cooper LS, Conwill $D E$, Clegg $L$, et al. Trends in the incidence of myocardial infarction and in mortality due to coronary heart disease, 1987 to 1994. N Eng J Med 1998;339(13):861-7.

11. Lotufo PA. Mortalidade precoce por doenças do coração no Brasil. Comparação com outros países. Arq Bras Cardiol 1998;70(5):321-5.
12. Gu K, Cowie CC, Harris MI. Diabetes and decline in heart disease mortality in US adults. JAMA 1999;281(16):1291-7.

13. Lakka HM, Laaksonen DE, Lakka TA, Niskanen LK, Kumpusalo $\mathrm{E}$, Tuomilehto J, et al. The metabolic syndrome and total and cardiovascular disease mortality in middle-aged men. JAMA 2002;288:2709-16.

14. Malik S, Wong ND, Franklin SS, Kamath TV, L'Italien GJ, Pio JR, et al. Impact of the metabolic syndrome on mortality from coronary heart disease, cardiovascular disease, and all causes in United States adults. Circulation 2004;110:1245-50.

15. Siqueira AFA, Franco LJ, Gimeno SGA, Matsumura LK, Barros Jr N, Ferreira SRG; on behalf of the JBDSG. Macrovascular disease in a Japanese-Brazilian population of high prevalence of metabolic syndrome: Associations with classical and non-classical risk factors. Atherosclerosis 2006;in press.

16. The Diabetes Control and Complications Trial/Epidemiology of Diabetes Interventions and Complications (DCCT/EDIC) Study Research Group. Intensive diabetes treatment and cardiovascular disease in patients with type 1 diabetes. $\mathbf{N}$ Eng J Med 2005;353:2643-53.

17. Laing SP, Swerdlow AJ, Slater SD, Burden AC, Morris A, Waugh NR, et al. Mortality from heart disease in a cohort of 23,000 patients with insulin-treated diabetes. Diabetologia 2003;46:760-5.

18. Laing SP, Swerdlow AJ, Carpenter LM, Slater SD, Burden AC, Botha $\mathrm{JL}$, et al. Mortality from cerebrovascular disease in a cohort of 23,000 patients with insulin-treated diabetes. Stroke 2003;34:418-21.

19. Yusuf S, Hawken S, Ounpuu S, Dans T, Avezum A, Lanas F, et al. Effect of potentially modifiable risk factors associated with myocardial infarction in 52 countries (the INTERHEART study): case-control study. Lancet 2004;364:937-52.

20. Hillary $H$, Aubert RE, Herman WH. Global burden of diabetes, 1995-2025. Prevalence, numerical estimates and projections. Diabetes Care 1998;21:1414-31.

21. Gross JL, Ferreira SRG, Oliveira JE. Glicemia pós-prandial. Arq Bras Endocrinol Metab 2003;47(6):728-38.

22. The Expert Committee on the Diagnosis and Classification of Diabetes Mellitus. Report of the Expert Committee on the Diagnosis and Classification of Diabetes Mellitus. Diabetes Care 2003;26(suppl.1):S5-20.

23. Harris R, Donahue K, Rathore SS, Frame P, Woolf SH, Lohr KN. Screening adults for type 2 diabetes: a review of the evidence for the U.S. Preventive Services Task Force. Ann Intern Med 2003;138:215-29.

24. The Expert Committee on the Diagnosis and Classification of Diabetes Mellitus. Follow-up report on the diagnosis of diabetes mellitus. Diabetes Care 2003;26:3160-7.

25. Harris MI, Flegal KM, Cowie CC, Eberhardt MS, Goldstein DE, Little RR, et al. Prevalence of diabetes, impaired fasting glucose, and impaired glucose tolerance in U.S. adults. The Third National Health and Nutrition Examination Survey, 1988-1994. Diabetes Care 1998;21:518-24.

26. Barrett-Connor E, Ferrara A. Isolated postchallenge hyperglycemia and the risk of fatal cardiovascular disease in older women and men: The Rancho Bernardo Study. Diabetes Care 1998;21:1236-9.

27. Saydah SH, Miret M, Sung J, Varas C, Gause D, Brancati FL. Postchallenge hyperglycemia and mortality in a national sample of U.S. adults. Diabetes Care 2001;24:1397-402.

28. The DECODE Study Group. Glucose tolerance and mortality: comparison of WHO and American Diabetes Association diagnostic criteria. Lancet 1999;354:617-21.

29. Meigs JB, Nathan DM, D'Agostino RB, Wilson PWF. Fasting and postchallenge glycemia and cardiovascular disease risk. The Framingham Offspring Study. Diabetes Care 2002;25:1845-50.

30. Klein R. Hyperglycemia and microvascular and macrovascular disease in diabetes. Diabetes Care 1995;18(2):258-68.

31. Laakso M. Hyperglycemia and cardiovascular disease in type 2 diabetes. Diabetes 1999;48:937-42.

32. Mather KJ, Verma S, Anderon TJ. Improved endothelial function with metformin in diet-treated type 2 diabetes mellitus. J Am Coll Cardiol 2001;37:1344-50. 
33. Quinones MJ, Hernandez-Pampaloni M, Schelbert H, BulnesEnriquez I, Jimenez X, Hernandez G, et al. Coronary vasomotor abnormalities in insulin-resistant individuals. Ann Int Med 2004; 140:700-8.

34. Nordt TK, Schneider DJ, Sobel BE. Augmentation of the synthesis of plasminogen activator inhibitor type-1 by precursors of insulin. A potential risk factor for vascular disease. Circulation 1994;89:321-30.

35. Lamarche B, Tchernof A, Mauriege $P$, Cantin B, Dagenais GR, Lupien PJ, et al. Fasting insulin and apolipoprotein B levels and low-density lipoprotein particle size as risk factors for ischemic heart disease. JAMA 1998;279:1955-61.

36. Boyne MS, Saudek CD. Effect of insulin therapy on macrovascular risk factors in type 2 diabetes. Diabetes Care 1999;22(3S):C45-53.

37. Kannel WB, Dawber TR, Kagan A, Revotskie N. Factors of risk in the development of coronary heart disease - six-year follow-up experience. The Framingham Study. Ann Intern Med 1961;55:33-50.

38. Wilson PW, D'Agostino RB, Levy D, Belanger AM, Silbershatz $\mathrm{H}$, Kannel WB. Find the total point score on the bottom table to determine the 10-year risk of CHD. Circulation 1998; $97: 1837-47$.

39. Folsom AR, Chambless LE, Ballantyne CM, Coresh J, Heiss G, Wu KK, et al. An assessment of incremental coronary risk prediction using $\mathrm{C}$-reactive protein and other novel risk markers: the atherosclerosis risk in communities study. Arch Intern Med 2006;166:1368-73.

40. Siqueira AFA, Abdalla, DSP, Ferreira SRG. LDL: da síndrome metabólica à instabilização da placa aterosclerótica. Arq Bras Endocrinol Metab 2006;50:334-43.

41. Executive Summary of The Third Report of The National Cholesterol Education Program (NCEP) Expert Panel on Detection, Evaluation, and Treatment of High Blood Cholesterol In Adults (Adult Treatment Panel III). JAMA 2001;285:2486-97.

42. Zambon A, Hokanson JE, Brown BG, Brunzell JD. Evidence for a new pathophysiological mechanism for coronary artery disease regression: hepatic lipase-mediated changes in LDL density. Circulation 1999;99(15):1959-64.

43. Steinberg D, Parthasarathy S, Carew TE, Khoo JC, Witztum JL. Beyond cholesterol. Modifications of low-density lipoprotein that increase its atherogenicity. N Engl J Med 1989;320(14):915-24.

44. Haffner SM, Stern MP, Hazuda HP, Mitchell BD, Patterson JK. Cardiovascular risk factors in confirmed prediabetic individuals. Does the clock for coronary heart disease start ticking before the onset of clinical diabetes? JAMA 1990;263:2893-8.

45. Lu W, Resnick HE, Jablonski KA, Jones KL, Jain AK, Howard WJ, et al. Non-HDL cholesterol as a predictor of cardiovascular disease in type 2 diabetes: The Strong Heart Study. Diabetes Care 2003;26:16-23.

46. Garg A, Grundy SM. Management of dyslipidemia in NIDDM. Diabetes Care 1990;13:153-69.

47. Pyorala K, Pedersen TR, Kjekshus J, Faergeman O, Olsson AG, Thorgeirsson G. Cholesterol lowering with simvastatin improves prognosis of diabetic patients with coronary heart disease. A subgroup analysis of the Scandinavian Simvastatin Survival Study (4S). Diabetes Care 1997;20:614-20.

48. Goldberg RB, Mellies MJ, Sacks FM, Moyé LA, Howard BV, Howard WJ, et al.; for the CARE Investigators. Cardiovascular events and their reduction with pravastatin in diabetic and glucose-intolerant myocardial infarction survivors with average cholesterol levels subgroup analyses in the Cholesterol And Recurrent Events (CARE) trial. Circulation 1998;98:2513-9.

49. Turner R, Holman R, Stratton I, Cull C, Frighi V, Manley S, et al. Tight blood pressure control and risk of macrovascular and microvascular complications in type 2 diabetes: UKPDS 38. BMJ 1998;317:703-13.

50. Adler Al, Stratton IM, Neil HAW, Yudkin JS, Matthews DR, Cull CA, et al.; on behalf of the UK Prospective Diabetes Study Group. Association of systolic blood pressure with macrovascular and microvascular complications of type 2 diabetes (UKPDS 36): prospective observational study. BMJ 2000;321:412-9.
51. Chobanian AV, Bakris GL, Black HL, Cushman WC, Green LA, Izzo, Jr JL, et al.; for the National High Blood Pressure Education Program Coordinating Committee. The Seventh Report of the Joint National Committee on Prevention, Detection, Evaluation, and Treatment of High Blood Pressure. JAMA 2003;289:2560-72.

52. HOPE Study. Effects of ramipril on cardiovascular and microvascular outcomes in people with diabetes mellitus: results of the HOPE study and MICRO-HOPE substudy. Heart Outcomes Prevention Evaluation Study Investigators. Lancet 2000;355:253-9.

53. Lindholm LH, Ibsen H, Dahlof B, Devereux RB, Beevers G, de Faire $U$, et al. Cardiovascular morbidity and mortality in patients with diabetes in the Losartan Intervention For Endpoint reduction in hypertension study (LIFE): a randomised trial against atenolol. Lancet 2002;359:1004-10.

54. Kuller LH, Becker DJ, Drash AL. Insulin-dependent diabetes mellitus mortality: The risk of cigarette smoking. Circulation 1990;82:37-43.

55. Al-Delaimy WK, Willett WC, Manson JE, Speizer FE, Hu FB. Smoking and mortality among women with type 2 diabetes. The Nurses' Health Study cohort. Diabetes Care 2001;24:2043-8.

56. Facchini FS, Hollenbeck CB, Jeppesen J, Chen YD, Reaven GM. Insulin resistance and cigarette smoking. Lancet 1992;339:1128-30.

57. Ajani UJ, Gaziano JM, Lotufo PA, Liu S, Hennekens CH, Buring JE, et al. Alcohol consumption and risk of coronary heart disease by diabetes status. Circulation 2000;102:500-5.

58. Gregg EW, Gerzoff RB, Caspersen CJ, Williamson DF, Narayan KMV. Relationship of walking to mortality among US adults with diabetes. Arch Intern Med 2003;163:14407.

59. Hu G, Eriksson J, Barengo NC, Lakka TA, Valle TT, Nissinen $A$, et al. Occupational, commuting, and leisure-time physical activity in relation to total and cardiovascular mortality among Finnish subjects with type 2 diabetes. Circulation 2004;110:666-73.

60. American Diabetes Association. Diabetic nephropathy. Diabetes Care 2003;26(S1):S94-8.

61. Mann JF, Gerstein HC, Pogue J, Bosch J, Yusuf S. Renal insufficiency as a predictor of cardiovascular outcomes. Diabetes Care 2000;23:B35-9.

62. Gerstein HC, Mann JFE, Yi O, Zinman B, Dinneen SF, Hoogwerf $B$, et al.; for the HOPE study investigators. Albuminuria and risk of cardiovascular events, death, and heart failure in diabetic and nondiabetic individuals. JAMA 2001;286:421-6.

63. Karalliedde J, Viberti G. Microalbuminuria and cardiovascular risk. Am J Hypertens 2004;17:986-93.

64. Rosenbaum P, Gimeno SG, Sanudo A, Franco LJ, Ferreira SRG. Independent impact of glycemia and blood pressure in albuminuria on high-risk subjects for metabolic syndrome. Clin Nephrol 2004;61:369-76.

65. Wachtell K, Ibsen H, Olsen MH, Borch-Johnsen K, Lindholm $\mathrm{LH}$, Mogensen CE, et al. Albuminuria and cardiovascular risk in hypertensive patients with left ventricular hypertrophy: The LIFE Study. Ann Intern Med 2003;139:901-6.

66. Alberti KG, Zimmet $P$, Shaw J. Metabolic syndrome - a new world-wide definition. A consensus statement from the International Diabetes Federation. Diabet Med 2006; 23:469-80.

67. Mohamed-Ali V, Goodrick S, Rawesh A, Katz DR, Miles JM, Yudkin JS, et al. Subcutaneous adipose tissue releases interleukin-6, but not tumor necrosis factor-alfa, in vivo. J Clin Endocrinol Metab 1997;82:4196-200.

68. Visser M, Bouter LM, McQuillan GM, Wener MH, Harris TB. Elevated C-reactive protein levels in overweight and obese adults. JAMA 1999;282:2131-5.

69. Ross R. Atherosclerosis - an inflammatory disease. N Engl J Med 1999;340:115-26.

70. Ridker PM, Rifai N, Stampfer MJ, Hannekens CH. Plasma concentration of interleukin- 6 and the risk of future myocardial infarction among apparently healthy men. Circulation 2000;101:1767-72. 
71. Ridker PM, Cushman M, Stampfer MJ, Tracy RP, Hennekens $\mathrm{CH}$. Plasma concentration of C-reactive protein and the risk of developing peripheral vascular disease. Circulation 1998; $97: 425-8$

72. Koening W, Sund M, Frohlich M, Fischer H, Lowel H, Doring $A$, et al. C-reactive protein, a sensitive marker of inflammation, predicts future risk of coronary heart disease in initially healthy middle-aged men: Results from the MONICA (Monitoring Trends and Determinants in Cardiovascular Disease) Augsburg Cohort Study, 1984 to 1992. Circulation 1999;99:237-42.

73. Albert CM, Jing Ma, Rifai N, Stampfer MJ, Ridker PM. Prospective study of C-reactive protein, homocysteine, and plasma lipid levels as predictors of sudden cardiac death. Circulation 2002;105:2595-9.

74. Ridker PM, Hennekens CH, Buring JE, Rifai N. C-reactive protein and other markers of inflammation in the prediction of cardiovascular disease in women. N Engl J Med 2000;342:836-43.

75. Ridker PM, Rifai N, Rose L, Buring JE, Cook NR. Comparison of C-reactive protein and low-density lipoprotein cholesterol levels in the prediction of first cardiovascular events. N Engl J Med 2002;347:1557-65.

76. Best JG, Zhang Y, Lee ET, Yeh J, Cowan L, Palmieri V, et al. $\mathrm{C}$-reactive protein as a predictor of cardiovascular risk in a population with a high prevalence of diabetes. The Strong Heart Study. Circulation 2005;112:1289-95.

77. Redberg RF, Greenland P, Fuster V, Pyörälä K, Blair SN, Folsom AR, et al. Prevention Conference VI: Diabetes and cardiovascular disease. Writing group III: Risk assessment in persons with diabetes. Circulation 2002;105:e144.

78. Grundy SM, Benjamin IJ, Burke JL, Chait A, Eckel RH, Howard $\mathrm{BV}$, et al. Diabetes and cardiovascular disease a statement for healthcare professionals from the American Heart Association. Circulation 1999;100:1134-46.

79. American Diabetes Association: Consensus development conference on the diagnosis of coronary artery disease in people with diabetes (Consensus Statement). Diabetes Care 1998;2:1551-9.

80. Gibbons LW, Mitchell TL, Wei M, Blair SN, Cooper KH. Maximal exercise test as a predictor of risk for mortality from coronary heart disease in asymptomatic men. Am J Cardiol 2000;86:53-8.

81. Laukkanen JA, Kurl S, Lakka TA, Tuomainen TP, Rauramaa R, Salonen $\mathrm{Rl}$, et al. Exercise-induced silent myocardial ischemia and coronary morbidity and mortality in middleaged men. J Am Coll Cardiol 2001;38:72-9.

82. O'Rourke RA, Brundage BH, Froelicher VF, Greenland $P$, Grundy SM, Hachamovitch R, et al. American College of Cardiology/American Heart Association Expert Consensus document on electron-beam computed tomography for the diagnosis and prognosis of coronary artery disease. Circulation 2000;102:126-40.

83. Haberl R, Becker A, Leber A, Knez A, Becker C, Lang C, et al. Correlation of coronary calcification and angiographically documented stenoses in patients with suspected coronary artery disease: results of 1,764 patients. J Am Coll Cardiol 2001;37:451-7.
84. Pletcher MJ, Tice JA, Pignone M, Browner WS. Using the coronary artery calcium score to predict coronary heart disease events. A systematic review and meta-analysis. Arch Intern Med 2004;164:1285-92.

85. Can U, Furie KL, Suwanwela N, Southern JF, Macdonald NR, Ogilvy CS, et al. Transcranila doppler ultrasound criteria for hemodynamically significant internal carotid artery stenosis based on residual lumen diameter calculated from en bloc carotid endarterectomy specimens. Stroke 1997;28:196671.

86. Hankey GJ, Warlow CP, Sellar RJ. Cerebral angiographic risk in mild cerebrovascular disease. Stroke 1990;21:209-22.

87. Beckman JA, Higgins CO, Gerhard-Herman M. Automated oscillometric determination of the ankle-brachial index provides accuracy necessary for office practice. Hypertension 2006; 47:35-38.

88. Sacks D, Bakal CW, Beatty PT, Becker GJ, Cardella JF, Raabe $\mathrm{RD}$, et al. Position statement on the use of the ankle brachial index in the evaluation of patients with peripheral vascular disease. A Consensus statement developed by the Standards Division of the Society of Interventional Radiology. J Vasc Interv Radiol 2003;14:S389.

89. UK Prospective Diabetes Study (UKPDS) Group. Intensive blood-glucose control with sulphonylureas or insulin compared with conventional treatment and risk of complications in patients with type 2 diabetes (UKPDS 33). Lancet 1998;352:837-53.

90. UK Prospective Diabetes Study (UKPDS) Group. Effect of intensive blood-glucose control with metformin on complications in overweight patients with type 2 diabetes (UKPDS 34). Lancet 1998;352(9131):854-65.

91. Gæde P, Vedel P, Larsen N, Jensen GVH, Parving H, Pedersen $O$. Multifactorial intervention and cardiovascular disease in patients with type 2 diabetes. N Engl J Med 2003;348:38393.

92. Dormandy JA, Charbonnel B, Eckland DJ, Erdmann E, MassiBenedetti M, Moules IK, et al.; for the PROactive investigators. Secondary prevention of macrovascular events in patients with type 2 diabetes in the PROactive Study (PROspective pioglitAzone Clinical Trial In macroVascular Events): a randomised controlled trial. Lancet 2005;366(9493):1279-89.

Endereço para correspondência:

Sandra Roberta G. Ferreira

Departamento de Nutrição

Universidade de São Paulo

Av. Dr. Arnaldo 715

01246-904 São Paulo, SP

Fax: (1 1) 3061-7701

E-mail: ferreira@medprev.epm.br 